\title{
Le plan Ecophyto de réduction d'usage des pesticides en France : décryptage d'un échec et raisons d'espérer
}

\author{
Laurence Guichard $^{1, *}$, François Dedieu ${ }^{2}$, Marie-Hélène Jeuffroy ${ }^{1}$, Jean-Marc Meynard ${ }^{3}$, \\ Raymond Reau ${ }^{1}$ et Isabelle Savini ${ }^{4}$ \\ ${ }^{1}$ UMR Agronomie, INRA, AgroParisTech, Université Paris-Saclay, avenue Lucien-Bretignières, 78850 Thiverval-Grignon, France \\ ${ }^{2}$ UMR Lisis, INRA, CNRS, ESIEE Paris UPEM, Université Paris-Est, 77454 Marne-la-Vallée, France \\ ${ }^{3}$ UMR SAD-APT, INRA, AgroParisTech, Université Paris-Saclay, 78850 Thiverval-Grignon, France \\ ${ }^{4}$ Délégation à l'expertise, à la prospective et aux études (DEPE), INRA, 75007 Paris, France
}

\begin{abstract}
Résumé - Le plan national Ecophyto, lancé en 2008 par le gouvernement français, qui visait une réduction de l'usage des pesticides de $50 \%$ en dix ans, "si possible », est un échec : en effet, au cours des cinq premières années de son application, la consommation de ces produits a augmenté. Ce constat a conduit les pouvoirs publics à annoncer un plan Ecophyto 2, en cours de mise en place. Pour les auteurs de cet article (agronomes et sociologues), l'échec était prévisible, au vu des caractéristiques des actions mises en place. Ils le montrent par l'analyse de deux actions phares du plan (le Bulletin de santé du végétal, base de l'information diffusée pour évaluer en temps réel les risques de bioagresseurs, et le réseau DEPHY de fermes de démonstration, conçu pour expérimenter et déployer des techniques économes en produits phytosanitaires), et l'analyse de l'usage fait des indicateurs de suivi du plan. Mais l'échec est imputable, plus encore peut-être, au fait que les actions n'ont ciblé que les agriculteurs et leurs conseillers, sans tenir compte des effets de « verrouillage sociotechnique », c'est-à-dire des interdépendances qui relient l'ensemble des acteurs économiques engagés dans la logique de systèmes agricoles pour lesquels les pesticides jouent un rôle de pivot. Le plan Ecophyto a cependant envoyé un signal symbolique fort, qui peut être déterminant à moyen et long termes : les pouvoirs publics annoncent clairement la fin de l'usage massif des pesticides en agriculture.
\end{abstract}

Mots clés : pesticide / indicateur / système de culture / politiques de l'environnement / système socioéconomique

\begin{abstract}
Ecophyto, the French action plan to reduce pesticide use: a failure analyses and reasons for hoping. Launched in France in 2008, the national "Ecophyto" plan, aiming "if possible" at a reduction of $50 \%$ of the pesticides use within 10 years, is a failure. After the first five years of its implementation, pesticide consumption has increased. Acknowledging this failure, the French government recently announced a new version of the plan (Ecophyto 2) although some aspects of its content remain unknown. For the authors of this paper (agronomists and sociologists), failure was predictable, given the characteristics of Ecophyto 1's implemented actions. They show it by the analysis of two of the flagship initiatives of the plan (monitoring health plant "bulletin", database allowing to assess in real time the risks of pests, and the "DEPHY farms", an experimental network supposed to disseminate good practices, and the study of the indicator measuring pesticide use. But the failure is even more due to the plan's main focus on farmers and advisors practices regardless of the broader effects of the "socio-technical lock-in" including a wide range of actors all interdependent and strongly engaged in pesticides' logic of uses. Ecophyto plan has nevertheless a symbolic effect, which could be determinant at mid- and long-terms: public authorities sent the signal of the upcoming end of pesticide massive use in agriculture.
\end{abstract}

Keywords: pesticide / indicator / cropping system / environmental policies / socioeconomic organization

\footnotetext{
* Auteur de correspondance : Laurence.Guichard@inra.fr
} 
Au cours des années 2000, différents rapports d'expertise (CPP, 2002 ; Momas et al., 2004 ; Aubertot et al., 2005) ont pointé les effets délétères des pesticides sur la santé des utilisateurs et sur l'environnement. Le Plan national santé environnement 2004 2008 a marqué, en France, un tournant dans la lutte contre les pollutions ayant un impact sanitaire, avec la création de l'Observatoire des résidus de pesticides. Les conséquences de l'utilisation massive de ces produits sur la perte de leur efficacité, liée à l'apparition de résistances chez les bioagresseurs ou encore le coût, pour l'agriculture, de ces intrants, ont également été soulignées. Ces éléments ont conduit à une forte volonté des pouvoirs publics de réduire l'utilisation de ces produits.

En 2006, la Commission européenne a adopté une stratégie thématique concernant l'utilisation durable des pesticides et a présenté une proposition de directive, qui prévoyait que les États membres mettent en œuvre des plans d'action nationaux pour atteindre cet objectif (directive définitive 2009/128/CE). Dans cette perspective, renforcée par le Grenelle de l'environnement de 2007, le gouvernement français a lancé en 2008 le plan « Ecophyto 2018 ». Associant la plupart des parties prenantes à sa mise en œuvre (organismes scientifiques et techniques, syndicats professionnels agricoles, entreprises productrices et distributrices de produits phytosanitaires, associations environnementales), ce plan visait, à échéance de dix ans, une réduction de l'usage des produits phytosanitaires de $50 \%$ « si possible », par rapport à la référence de consommation de la campagne 2008. Depuis, malgré des moyens engagés importants, les résultats sont pour le moins décevants : sur la période 2009-2013, l'indicateur retenu pour le suivi du plan (le nombre de doses unités [NODU], $c f$. infra) enregistre une augmentation de la consommation nationale de pesticides de $5 \%$. En 2014, la mission confiée par le premier ministre « en vue de proposer une nouvelle version du plan Ecophyto » entérine l'échec du premier plan et formule des recommandations pour le suivant (Potier, 2014). S'appuyant pour partie sur ce rapport, une première version du plan «Ecophyto 2 » est rendue publique par les ministères en charge de l'agriculture et de l'environnement fin octobre 2015.

L'objectif de cet article est d'éclairer les principales raisons de cet échec du plan Ecophyto 1. Nous nous appuyons sur différents rapports et études officiels réalisés sur la période : le rapport Potier (2014) et les évaluations à mi-parcours des actions majeures du plan. Nous mobilisons également les recherches que nous conduisons, en agronomie des grandes cultures et en sociologie, et notamment une quarantaine d'entretiens semidirectifs approfondis avec l'ensemble des acteurs impliqués dans les négociations du Grenelle de l'environnement, réalisés en 2008, ainsi qu'une enquête sur l'organisation de la filière arboricole menée en 2015-2016 auprès de 53 arboriculteurs. Nous nous référons aussi à notre participation à plusieurs opérations ou dispositifs qui ont préparé ou accompagné le premier plan : les expertises «Pesticides » (Aubertot et al., 2005) puis « Ecophyto R\&D » (Butault et al., 2010 ; Jacquet et al., 2011), ainsi que différentes instances de pilotage ou de suivi de certaines actions du plan.

\section{Le plan Ecophyto en bref}

Le plan Ecophyto 1 est piloté par la direction générale de l'alimentation (DGAl) du ministère en charge de l'agriculture. Il est financé par la redevance pour pollution diffuse (RPD, prélevée auprès des distributeurs de produits phytosanitaires), complétée de crédits d'État (programmes ministériels des administrations) et de crédits issus des autres parties prenantes du plan (autofinancement des partenaires, collectivités territoriales, fonds de formation, programmes de recherche...). Sur la période 2009-2014, il a bénéficié de 361 millions d'euros (M€) de financement, dont $194 \mathrm{M} €$ issus de la redevance (Potier, 2014). L'affectation de ces moyens aux différents axes du plan n'est connue (Fig. 1) que pour les crédits issus de la RPD (plafonnés depuis 2012 à 41 M€ par an).

Parmi les actions considérées comme majeures figure le réseau DEPHY, composé de fermes de démonstration et d'acquisition de références, ainsi que d'expérimentations de systèmes de culture économes en produits phytosanitaires. Couvrant l'ensemble des grandes filières de production françaises, le réseau comptait en 2015 près de 190 groupes d'une dizaine d'exploitations agricoles, animés chacun par un ingénieur réseau à mi-temps. Le soutien à cette action pour les réalisations 2009-2014 en fait l'un des postes les plus consommateurs des financements (Fig. 1).

La seconde action majeure, par le budget alloué sur la période 2009-2014, est la création du Réseau de surveillance biologique du territoire. Il comprenait, en 2014, 15400 parcelles agricoles sur lesquelles la présence de maladies et ravageurs était régulièrement suivie pendant la campagne agricole par environ 4000 observateurs (issus essentiellement des organismes de R\&D et des coopératives et négoces). Ces suivis donnent lieu à la publication hebdomadaire de «bulletins de santé du végétal » (BSV) sur l'ensemble des régions (3 500 BSV publiés en 2014) informant les agriculteurs et leurs conseillers de l'état sanitaire des cultures, afin de les aider à mieux cibler leurs interventions.

Parmi les autres actions significatives figurent la mobilisation des établissements d'enseignement agricole pour compléter le réseau DEPHY par un volet pédagogique, ou encore la formation des professionnels (distributeurs, conseillers et applicateurs) à une utilisation sécurisée des produits phytosanitaires pour maîtriser les risques pour la santé publique et l'environnement, sanctionnée par l'obtention du Certiphyto. Des actions ont porté sur la production et la diffusion d'outils et de connaissances, avec la rédaction de " guides de coconception de systèmes économes en produits phytosanitaires ॥ pour les principales filières (grandes cultures, marâ̂chage, vigne...), et la création en 2012 d'Ecophyto PIC, le portail informatique documentaire de la protection intégrée des cultures. Notons enfin que le volet « Recherche » du plan («R\&D» sur la Fig. 1) n'a pas fait partie des actions significatives au regard des crédits RPD attribués (12 M€ sur la période, moins que pour l'axe communication). Mais il a été notamment conforté par des financements propres d'autres appels à projets existants sur des recherches spécifiques dédiées aux pesticides et répondant aux objectifs du plan (Ricci, 2015).

\section{Les objectifs du plan et les outils de suivi}

\subsection{Des objectifs très ambitieux, peu fédérateurs}

Le plan a d'abord défini sur quoi devait porter la réduction : l'utilisation des pesticides ou les (risques d') impacts? L'expertise scientifique collective (ESCo) 


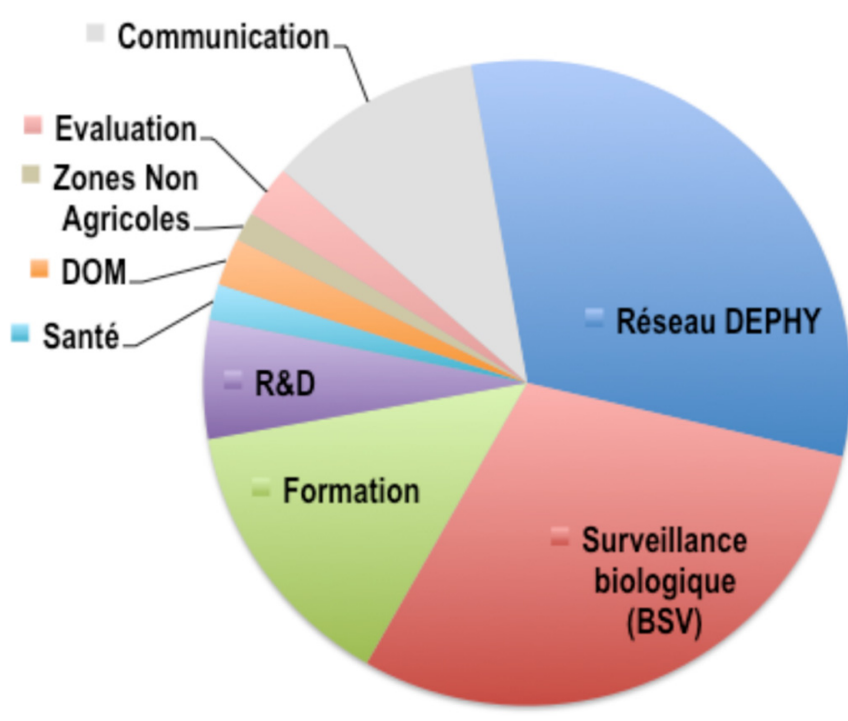

Fig. 1. Répartition des financements attribués sur la période 20092014 (194 M€) aux principaux programmes du plan Ecophyto à partir de la dotation nationale de RPD. Les réseaux de fermes DEPHY et la surveillance biologique du territoire sont les postes les plus consommateurs de financement, devant la formation Certiphyto (" formation »), la communication et la recherche ("R\&D") (Potier, 2014).

Fig. 1. Distribution of fundings from the diffuse pollution fee allocated during 2009-2014 (194 M€) to main Ecophyto plan programs. DEPHY farms (réseau DEPHY) and pests monitoring (surveillance biologique) networks are the biggest fundings consumers, before the Certiphyto training ("formation"), the communication ("communication") and the research ("R\&D”) (Potier, 2014).

« Pesticides » (Aubertot et al., 2005) affirmait, dès son titre, la nécessité de réduire l'utilisation des pesticides pour réduire leurs impacts. Au travers du plan interministériel de réduction des risques liés aux pesticides (2006-2009) et de son axe 2, « agir sur les pratiques et minimiser le recours aux pesticides ", le ministère en charge de l'agriculture a soutenu le développement de systèmes de culture économes en pesticides. S'appuyant sur l'ESCo « Pesticides », il y voyait une réponse aux enjeux environnementaux, mais aussi un atout à moyen terme pour l'agriculture : rendre les systèmes plus économes, et donc moins dépendants de l'usage des pesticides, était aussi un moyen de réduire l'exposition des opérateurs et de limiter les risques d'apparition de résistances dans un contexte de diminution du nombre de substances actives (SA) autorisées (moins de $50 \%$ en 20 ans).

Dans sa version initiale de septembre 2008, intitulée « plan Ecophyto 2018 de réduction des usages de pesticides », le plan a donc bien retenu un objectif de baisse des consommations nationales de pesticides, et un indicateur de suivi adapté, fondé sur les ventes de produits phytosanitaires ( $c f$. infra).

Le second élément important du plan a été la fixation de l'objectif quantitatif de réduction. L'objectif d'une baisse de $50 \%$ a émergé lors des débats du Grenelle de l'environnement, porté par les associations environnementalistes, comme l'illustre l'extrait de l'entretien ci-dessous :
«Au départ on pensait que moins $50 \%$ c'était totalement irréaliste, on pensait que cela ne passerait jamais. [...] Mais on s'est rendu compte que, finalement, l'objectif d'une baisse de moitié ne faisait pas sauter au plafond les interlocuteurs, voire même ne rencontrait pas d'opposition franche. [...] Comme on a vu que France Nature Environnement proposait une baisse de moitié, on a décidé de s'aligner et c'est devenu notre position commune. » (Générations Futures, entretien du 13 octobre 2009).

En janvier 2010, l'étude Ecophyto R\&D (Butault et al., 2010), à laquelle ont participé 96 experts issus de plus de 30 organismes, concluait à la faisabilité d'une baisse de $30 \%$ sans perte significative de production ni bouleversement des systèmes de production, le résultat pouvant être obtenu par un développement de modes de conduite raisonnés et d'itinéraires techniques « bas intrants ».

Le premier plan portait donc un objectif ambitieux de réduction des utilisations (moins de $50 \%$, si possible), qui, d'après l'étude Ecophyto R\&D (Jacquet et al., 2011), nécessitait d'engager des actions allant bien au-delà de l'optimisation «classique » des pratiques agricoles pour favoriser l'efficience des intrants utilisés, c'est-à-dire de reconcevoir les systèmes pour créer des conditions défavorables au développement des bioagresseurs des cultures (Hill et McRae, 1995) (Encadré 1). Par sa cible (les usages) et son cap ambitieux, le plan Ecophyto 1 a très tôt été remis en cause par la plupart des acteurs de la R\&D et des filières. Ceux-ci ont exprimé, dans les «cahiers d'acteurs de l'étude Ecophyto $\mathrm{R} \& \mathrm{D} \gg(2010)$, des réserves sur les références mobilisées dans l'étude, des doutes sur la possibilité de réduire fortement les pesticides sans perte économique, et enfin une nette préférence pour la généralisation de travaux sur le raisonnement et l'optimisation de la lutte chimique via les outils d'aide à la décision (cahiers d'acteurs Coop de France, ACTA, Arvalis, CTIFL et UIPP). La réduction permise par le savoir-faire disponible, selon Coop de France, se situait autour de 15 à $20 \%$.

Encadré 1 Les moyens de réduire l'usage des pesticides. De nombreuses alternatives aux pesticides existent. Comme le montrent tant la littérature scientifique (Aubertot et al., 2005 ; Gliessman, 2007 ; Loyce et al., 2012 ; Deguine et al., 2016) que les pratiques d'agriculteurs utilisant peu de pesticides (Petit et al., 2012 ; Butault et al., 2010), les techniques permettant de réduire les populations de bioagresseurs sont nombreuses : variétés résistantes aux maladies, associations de variétés ou d'espèces, successions culturales et assolements diversifiés, désherbage mécanique, lutte biologique par lâchers d'auxiliaires exogènes ou aménagements paysagers favorisant les auxiliaires indigènes, confusion sexuelle... La plupart de ces techniques, prises isolément, ne sont pas suffisantes pour contenir les épidémies et éviter tout dégât, mais en les combinant, on peut souvent atteindre des efficacités comparables à celle des pesticides. 
La vision centrée sur une réduction ambitieuse des usages a, depuis, toujours fait l'objet de protestations des instances agricoles. Par exemple Philippe Pinta, président d'ORAMA (union fédérant les associations spécialisées des producteurs de blé, de maïs et d'oléagineux et protéagineux de la Fédération nationale des syndicats d'exploitants agricoles [FNSEA]), écrit ainsi en 2015 (Pinta, 2015) : «Tel que continuent à le concevoir certains ministres en voulant précipiter les choses et imposer des objectifs et mesures irréalistes, Ecophyto 2, c'est de la politique politicienne ! $50 \%$ de baisse des phytos d'ici 2025 s'il n'y a pas de solution de remplacement, c'est de l'incantation, de la démagogie. Fi de nos aptitudes et contraintes, de l'équilibre de nos entreprises, du dynamisme de nos filières, de tout ce que notre secteur apporte au pays ! Autant de mépris ne peut que mener à l'impasse. C'est d'une irresponsabilité frappante. »

\subsection{Des indicateurs de réussite non dénués d'ambiguïtés}

Le plan Ecophyto 1 affichant un objectif de réduction de l'utilisation des pesticides, les principaux indicateurs retenus pour son suivi ont bien porté sur cet usage : ce sont, au niveau national, la quantité de substances actives (QSA), le NODU et l'indicateur de fréquence de traitement (IFT) (Encadré 2).

Conçu comme indicateur de la « dépendance » des modes de production aux pesticides (qu'il s'agissait de réduire), l'IFT semble aujourd'hui l'objet d'un certain mésusage. En effet, pour certains acteurs, la baisse de l'IFT est devenue une fin en soi, qu'il est facile d'atteindre si l'on connaît son mode de calcul. Nous avons observé, dans différentes régions, des tactiques de recherche d'une baisse de l'IFT par l'emploi de produits qui comptent moins de points d'IFT pour une même efficacité biocide. Ainsi, l'application de produits commerciaux contenant plusieurs SA est plus favorable que l'emploi d'une association équivalente de produits monosubstances (par exemple, deux programmes anti-mildiou de la vigne, l'un utilisant un produit pré-mélangé à base de deux SA, l'autre apportant ces mêmes SA par l'application de deux produits simples, comptent respectivement pour 1 et 1,8 points d'IFT, alors qu'ils sont équivalents). Ces tactiques, bien connues des conseillers agricoles, conduisent à des baisses artificielles d'IFT, ne correspondant à aucune réduction du recours aux pesticides. L'utilisation de l'IFT comme indicateur de résultat dans la mesure agri-environnementale (MAE) « Phyto » (qui engage l'agriculteur à réduire, en cinq ans, l'utilisation des pesticides) est certainement en partie à l'origine de cette dérive, même si, de fait, elle a également contribué à largement faire connaître et déployer l'IFT dans le monde agricole, audelà des seules MAE. Pour éviter ces biais, les ministères en charge de l'environnement et de l'agriculture ont confié à l'INRA la mise au point de l'IFT «SA », calculé non plus sur les produits commerciaux, mais sur les SA (Le Breton, 2014). Mais plus de deux ans après la conception de ce nouveau mode de calcul de l'IFT, celui-ci n'a pas encore été mis en œuvre par les ministères, notamment par manque de moyens humains pour en assurer la gestion.

Le NODU n'est pas exposé aux mêmes risques de dérive : calculé par les services de l'État à l'échelle nationale sur les données de vente, il n'est pas utilisé directement par les acteurs
Encadré 2 Les indicateurs centraux du plan : quantité de substances actives (QSA), indicateur de fréquence de traitement (IFT) et nombre de doses unités (NODU).

La QSA est l'indicateur « historique ». Largement utilisé en Europe, il évalue la consommation de pesticides par les quantités vendues sur un territoire (aujourd'hui déclarées annuellement par les distributeurs en France dans le cadre de la redevance pour pollution diffuse). L'ESCo Pesticides (2005) pointait ses limites : la mise sur le marché de molécules actives efficaces à des doses toujours plus faibles pour une même action biocide induit mécaniquement une baisse des tonnages qui ne correspond à aucune réduction de la dépendance de l'agriculture à ces produits.

L'IFT (calculé sur les pratiques déclarées des agriculteurs) a été adapté de l'indicateur danois (Gravesen, 2003), dans le cadre d'une étude confiée à l'INRA en 2006 par le ministère chargé de l'agriculture, pour évaluer le degré de dépendance des pratiques agricoles à l'utilisation de pesticides. Il est fondé sur une normalisation des produits commerciaux par leur dose d'homologation qui permet de sommer dans un même programme des produits aux caractéristiques très différentes et ainsi éviter l'écueil de la QSA. Le point d'IFT s'interprète comme un «proxy » d'unité d'activité biocide. Une parcelle à 4 points d'IFT est considérée comme deux fois plus dépendante des pesticides qu'une parcelle à 2 points (le « besoin » de traiter est double). Cet indicateur est aujourd'hui largement utilisé dans le réseau DEPHY où il est calculé à la fois par culture et à l'échelle pluriannuelle de la succession des cultures.

Le NODU (calculé à partir des déclarations annuelles des ventes de pesticides par les distributeurs) est dérivé de l'IFT. Il repose sur la même logique de normalisation, mais appliquée aux SA, ce qui permet de sommer des substances employées à des doses très différentes. Le NODU est calculé annuellement, à l'échelle de la ferme France.

de terrain, et il est en conséquence plus robuste vis-à-vis des risques de mésusages. Ses valeurs ont augmenté sur cinq ans, ce qui renforce la réaction d'acteurs visant à délégitimer l'objectif initial de réduction d'usage pour (re)mettre en avant un objectif d'impact (souvent réduit au compartiment eau). Le site internet du groupe coopératif InVivo souligne ainsi : «L'efficacité du nouveau plan Ecophyto ne peut se mesurer sur la seule base d'indicateurs quantitatifs relatifs à la baisse des usages des produits phytopharmaceutiques (NODU). InVivo privilégie pour sa part des indicateurs mesurant la réduction des impacts environnementaux et risques sanitaires des pratiques agricoles en phase en cela avec la réglementation européenne. " (InVivo, 2015).

Aujourd'hui, la version 2 du plan annonce la mise en œuvre d'un nouveau « panier d'indicateurs » qui viendrait compléter le NODU. Ce panier comprendrait quatre indicateurs renseignant :

- la comptabilisation des «bonnes pratiques et solutions innovantes » reconnues dans le cadre du dispositif des 
Certificats d'économie de produits phytopharmaceutiques (CEPP, dispositif d'incitation des distributeurs de produits phytosanitaires à promouvoir ou à mettre en œuvre auprès des utilisateurs professionnels des actions permettant d'en réduire l'utilisation, les risques et les impacts) et publiées par un arrêté du ministère en charge de l'agriculture ;

- la comptabilisation de la diffusion de ces solutions et de l'accompagnement des agriculteurs ;

- l'estimation de l'adoption des bonnes pratiques par les agriculteurs ;

- la quantification de l'évolution de l'utilisation des produits phytosanitaires au travers du rapport QSA/SAU (surface agricole utile).

Cette évolution acte une voie nouvelle pour le plan : celle de déplacer la question, jusqu'ici considérée comme centrale, du résultat en termes de dépendance vis-à-vis des pesticides (mesurée par un indicateur de résultats, le NODU), vers la question des moyens mis en œuvre (les CEPP et plus largement les «bonnes pratiques») qui ne garantissent en rien l'évolution des consommations.

\section{La logique et la mise en œuvre des deux actions «phares » du plan}

Premières actions mises en œuvre, et bénéficiant de l'essentiel des financements issus de la redevance alloués au plan, le BSV et le réseau de fermes DEPHY sont bien les dispositifs phares du plan Ecophyto 1. Ces deux actions ont fait l'objet d'évaluations in itinere conduites par des bureaux d'études (Blanchet, 2012) et pilotées par le ministère chargé de l'agriculture (Dreyfus, 2014 pour le CGAER).

\subsection{Le BSV : un dispositif « recyclé »}

Historiquement, la surveillance des maladies et ravageurs des cultures était une mission du ministère de l'Agriculture. Dès 1941, ses services régionaux de la protection des végétaux ont produit et diffusé des avertissements agricoles sur l'état sanitaire des cultures. Mais progressivement, de nombreux acteurs locaux, parapublics (chambres d'agriculture) ou privés (coopératives, organisations de producteurs...), ont intégré cette fonction de surveillance des cultures dans leur activité de conseil. La sous-direction de la qualité et de la protection des végétaux (SDQPV) s'est appuyée sur ce constat pour envisager, dès 2005, de déléguer cette compétence à ces acteurs territoriaux. Elle entendait ainsi répondre aux objectifs de baisse des dépenses publiques, associés à la révision générale des politiques publiques.

En 2008, lors de la conception du plan Ecophyto 1, la SDQPV a ainsi vu l'opportunité d'y inclure son projet de réforme de la surveillance des bioagresseurs, justifié par l'argument que la surveillance est une stratégie efficace pour raisonner l'usage des pesticides. Le projet a été retenu et $5 \mathrm{M} €$, sur les 9 alloués initialement au plan, ont été affectés au BSV en 2009. Cet important budget a permis de financer le volet le plus onéreux du projet : le recrutement des animateurs locaux chargés de coordonner les futurs réseaux de surveillance implantés sur le territoire.
La priorité donnée au BSV dans le plan Ecophyto a donc indéniablement obéi à des considérations stratégiques. Mais ce dispositif a aussi été soutenu parce qu'il correspond le mieux à l'idée que se fait le ministère d'un usage raisonné des produits phytosanitaires : « surveiller pour traiter au plus juste » reste, selon les agents de la SDQPV, le meilleur moyen de concilier les objectifs de réduction d'usage des pesticides avec la sécurisation des cultures - leur mission première - qui passe toujours à l'heure actuelle par les traitements chimiques. Or cette logique, caractéristique de l'agriculture raisonnée, n'a jamais été pensée pour réduire l'usage des pesticides de $50 \%$. Ainsi, un agent du SDQPV interrogé explique : « Il ne faut pas oublier que ce qui nous intéresse, c'est la santé des plantes. On essaye de faire au mieux et de prendre en compte les exigences sociétales vis-à-vis des pesticides, mais malheureusement pour le moment on n'a pas d'autres choix que d'utiliser les pesticides. Est-ce qu'il existe des traitements non chimiques pour lutter contre toutes les maladies sur des hectares aussi importants que l'on peut avoir en grandes cultures par exemple ? » (entretien du 24 novembre 2009).

Au-delà de sa genèse politique, les caractéristiques du BSV permettent-elles d'en faire un outil utile à l'objectif de réduction d'utilisation des pesticides porté par le plan Ecophyto ? L'évaluation du CGAAER (conseil de l'alimentation) constate en 2012 qu'il est délicat d'apprécier l'efficacité du BSV : « L'évaluation peut conclure sur la notoriété du BSV et son intérêt pour les conseillers et les agriculteurs enquêtés ( $80 \%$ des agriculteurs connaissent le BSV, $72 \%$ s'appuient dessus pour une décision ; $99 \%$ des conseillers consultent le BSV, $61 \%$ le citent dans leurs bulletins techniques). » Mais concernant la réduction d'usage des pesticides, l'étude in itinere « ne peut pas vraiment conclure. À ce jour, elle constate qu'un tiers des agriculteurs enquêtés déclarent que le BSV permet de réduire et/ou modifier les pratiques phytosanitaires » (Blanchet, 2012, p. 12).

L'analyse du contenu du BSV et du mode d'acquisition des données apporte un autre éclairage. Appuyé sur le suivi d'un réseau de "parcelles représentatives" qui assure une surveillance quantitative des maladies et ravageurs des cultures, le BSV diffuse une information "à chaud » sur la présence ou l'absence de ces bioagresseurs. Il fonctionne donc comme un système d'alerte, largement relayé par les organismes stockeurs via des messages SMS à leurs adhérents. Cette alerte peut éviter ou retarder les traitements inutiles à l'instant $t$. Mais elle peut aussi alarmer des agriculteurs, et les inciter à « sortir le pulvérisateur » sans toujours vérifier si le bioagresseur est présent sur leurs propres parcelles. Le media SMS, trop court pour apporter des nuances, expose particulièrement à ce risque, comme l'illustre cet exemple de SMS émis en mars 2016 par une coopérative de la région Centre-Val-de-Loire : « Nombreuses captures de charançons hier dans les colzas ; intervenir sous 4 à 5 jours ».

L'alerte est un outil qu'il faudrait pouvoir utiliser dans les deux sens : pour inciter à ne pas traiter dans certaines situations, comme à traiter dans d'autres parcelles. Pour répondre aux enjeux de la réduction d'usage, il serait important de décrire les différentes situations de culture et de pression biotique associée, pour différencier les recommandations suivant ces situations. Or, non seulement les BSV actuels s'interdisent d'aller jusqu'à des recommandations, mais l'absence de diagnostic approfondi sur les écarts entre 
situations empêche les utilisateurs de comprendre les mécanismes en jeu, les interactions entre combinaison de pratiques et pression biotique, et nuisent à leur apprentissage des pratiques économes en pesticides. Faire du BSV un outil au service de la réduction d'usage impliquerait un réseau de surveillance différent, dont l'échantillon de parcelles serait choisi pour explorer et suivre une diversité de situations agronomiques, à même d'induire des pressions de bioagresseurs différentes, qu'il viserait à expliquer. Ce n'est pas envisagé pour le moment par les pouvoirs publics.

Considéré comme action majeure du plan Ecophyto 1 (au moins par l'ampleur des crédits alloués), le BSV reste, pour les porteurs de sa version 2, une pierre angulaire qu'il s'agit de renforcer. Mais très peu d'informations sont à ce jour fournies sur les objectifs et la nature de ce renforcement. S'il conduit juste à accroître la taille du réseau des parcelles suivies (par une augmentation concomitante du financement lié à l'observation), sans développer conjointement cette culture diagnostique qui fait aujourd'hui cruellement défaut, le nouveau plan aidera-t-il vraiment à la transition vers des systèmes plus économes ?

\subsection{Le réseau de fermes DEPHY : un changement d'échelle impensé ?}

Sur la base des propositions issues de l'étude Ecophyto R\&D, commanditée par les ministères chargés de l'agriculture et de l'environnement en amont du plan Ecophyto 1, un premier réseau de 178 fermes a été initié en 2010 : sa "vocation [...] n'est pas de se limiter à un rôle de démonstration. C'est sur la qualité des références qui pourront y être produites et sur l'intérêt des systèmes de culture mis en œuvre, plus que sur la capacité du chef d'exploitation à communiquer, qu'il est important de sélectionner les exploitations » (Reau et Fortino, 2010). Comme le soulignent Petit et al. (2012) puis Dumas et al. (2012), ce premier réseau « test » avait un triple rôle :

- produire des références sur les systèmes de culture économes en produits phytosanitaires ;

- favoriser la transformation des systèmes de culture ;

- démontrer, communiquer, servir de support de formation.

Ce réseau s'est ensuite rapidement étendu à plus de 1900 fermes pilotes à partir de 2011, sous la responsabilité d'une nouvelle équipe, la Cellule d'animation nationale (CAN), gérée par l'Assemblée permanente des chambres d'agriculture (APCA). Il prend alors le nom de DEPHY pour Démonstration Expérimentation Production de références sur les systèmes économes en pHYtosanitaires. «Un axe majeur du plan Ecophyto est d'abord d'identifier, puis d'assurer la diffusion de ces pratiques innovantes et des systèmes de culture économes en pesticides », souligne la directrice de la DGAl au ministère de l'Agriculture, pilote du plan, dans un exposé sur les orientations et les objectifs du plan Ecophyto à l'Académie d'agriculture de France (Soubeyran, 2014). Or, depuis 2010, l'absence de baisse de l'IFT et du NODU au niveau national laisse penser que la diffusion des pratiques économes n'a pas fonctionné. Deux analyses, l'évaluation in itinere du dispositif DEPHY (TERCIA, 2014 ; Dreyfus, 2014) et une étude de trois groupes de fermes et du dispositif global de DEPHY (Pestimute-Gen, réalisée dans le cadre du programme de recherche Pour et Sur le Plan Ecophyto, volet « Recherche » du plan), fournissent des éléments d'explication, qui concernent trois points :

- les modalités de constitution du réseau ;

- les activités demandées aux animateurs des groupes de fermes ;

- l'absence de plan d'action stratégique.

\subsubsection{La constitution du réseau de fermes : des objectifs antagonistes}

Le réseau de fermes DEPHY développé à partir de 2011 a été constitué sur la base du volontariat. Il est d'une grande diversité, intégrant des agriculteurs déjà avancés ou engagés dans la mise en œuvre de pratiques économes en pesticides, mais aussi beaucoup d'agriculteurs ayant des pratiques très consommatrices. Ainsi, environ $66 \%$ des fermes DEPHY en grande culture étaient considérées comme peu ou pas économes à leur entrée dans le réseau (IFT initial supérieur à $70 \%$ de l'IFT de référence, qui correspond au septième décile régional). Ces valeurs étaient de $56 \%$ en polyculture/élevage, $62 \%$ en arboriculture et $67 \%$ en viticulture (Ecophyto, 2015). Or une réduction importante de l'usage des pesticides nécessite des changements profonds et pluriannuels dans les pratiques (Jacquet et al., 2011). De fait, en 2014, la part de systèmes qualifiés de « très économes » (IFT inférieur à $50 \%$ de l'IFT de référence) reste faible pour certains systèmes et peu évolutive : $15 \%$ en grande culture (soit $2 \%$ de plus qu'au démarrage du réseau), $18 \%$ en viticulture $(+6 \%), 38 \%$ en arboriculture $(+18 \%)$, et $40 \%$ en polyculture-élevage $(+13 \%)$. La production de références sur des systèmes économes aurait donc sans doute été plus convaincante et efficace si elle s'était appuyée sur une plus grande proportion de fermes déjà engagées dans cette voie et disposant de solutions éprouvées. Dès sa constitution, le réseau DEPHY a été tiraillé entre deux objectifs antagonistes : d'un côté, choisir des fermes ayant déjà réduit de manière importante leur usage de pesticides, en vue d'une analyse approfondie de leurs systèmes, pour fournir des références aux autres fermes; d'un autre côté, choisir des fermes dont la dépendance aux pesticides était élevée au début du plan, pour démontrer, assez rapidement, la possibilité de réduire cet usage. Cependant, pour que le second groupe de fermes réussisse de manière durable cette réduction, il aurait été nécessaire de disposer des références dont la production dépendait du premier groupe...

Pour garantir aux pouvoirs publics des preuves de succès d'un plan coûteux pour la France, quantifiables par le suivi de la valeur d'IFT, les responsables de la mise en place du réseau ont en définitive préféré centrer les fermes DEPHY sur l'objectif de baisse de leur propre IFT. L'objectif de production de références sur des systèmes très économes, dont l'efficacité technique ne se révèle que plusieurs années après l'introduction des changements (Mischler et al., 2009 ; Salembier et al., 2016), a été fragilisé par ce choix.

\subsubsection{L'évolution des activités demandées aux animateurs des groupes de fermes}

Dès 2011, la responsable de la CAN a mis l'accent sur la communication, via des journées de démonstration dans les fermes DEPHY : « La mise en œuvre par les exploitants du 
réseau [...] permet pour le monde associatif et le grand public, d'être informés des progrès de l'agriculture dans la prise en compte des enjeux de société » (Le Corre, 2011). Il apparaît que l'essentiel, pour les responsables de la mise en œuvre du plan, a été de communiquer sur les actions lors d'opérations à forte visibilité institutionnelle et sociétale, pour rassurer sur l'activité effective du dispositif et la justification des financements alloués, et pour susciter l'adhésion de nouveaux entrants (Cerf et al., 2015).

Viser une réduction de $50 \%$ de l'usage de pesticides exige, de la part des agriculteurs, une forte activité de conception pour mettre au point des systèmes de culture valorisant les conditions locales de production (régulations biologiques, mais aussi ressources logistiques, humaines et matérielles), comme l'a montré l'analyse des systèmes économes et performants du réseau test (Dumas et al., 2012). Dans cette activité de conception, qui ne peut être réduite à l'adoption de solutions ayant montré leur efficacité dans d'autres contextes, les agriculteurs ont souvent besoin d'être accompagnés, par des conseillers et/ou par des groupes de pairs (Mischler et al, 2009 ; Cerf et al., 2015). On peut se demander si l'importance donnée à la démonstration et à la communication n'a pas empêché les animateurs des groupes de se concentrer sur la connaissance des systèmes économes en pesticides éprouvés par les agriculteurs et l'accompagnement des agriculteurs dans leur mise au point.

Le lancement, à partir de 2013, de la base de données AGROSYST a certainement contribué à réorienter significativement l'activité des animateurs. En effet, au niveau national, les responsables du réseau DEPHY ont fait l'hypothèse que la mise à disposition, dans une base commune, des descriptifs techniques des systèmes de culture des agriculteurs engagés dans le réseau, était une étape clé pour identifier, à partir d'analyses statistiques, les solutions techniques les plus appropriées pour des systèmes économes et performants. Ce choix a exigé alors, de la part des animateurs des groupes, d'investir un temps non négligeable dans l'enregistrement des pratiques culturales des agriculteurs de leur groupe, au détriment du temps consacré à la conception et à la connaissance des systèmes, afin d'assurer une "remontée » rapide des données vers la $\mathrm{CAN}$, pour une analyse à l'échelle nationale (Pillet, 2014). Ainsi que le souligne l'évaluation du plan Ecophyto, « un mécanisme de déséquilibre entre le processus de construction du réseau et l'acquisition de données sur les exploitations membres des groupes s'est mis en place, au profit de cette dernière » (TERCIA, 2014).

Initialement vus comme des producteurs de ressources pour l'action et des accompagnateurs du changement, comme le proposaient Reau et Fortino (2010) dans l'étude Ecophyto R\&D, les animateurs des groupes DEPHY ont dû considérablement amplifier leur rôle de collecteurs de données, réduisant ainsi leur implication dans l'accompagnement des agriculteurs qui veulent faire évoluer leurs systèmes.

\subsubsection{L'absence de plan d'action stratégique pour une généralisation de pratiques économes}

Sur la base du bilan du plan Ecophyto 1, les ministères chargés de l'agriculture et de l'environnement ont souhaité, dès 2014, généraliser les résultats à l'ensemble des acteurs concernés à travers le lancement du plan Ecophyto 2: «Désormais le principal défi est de valoriser et de déployer auprès du plus grand nombre les techniques et systèmes économes et performants qui ont fait leurs preuves chez quelques-uns [...]. Le réseau DEPHY sera progressivement élargi à 3000 fermes [...]. La diffusion des pratiques innovantes économes en produits phytopharmaceutiques reposera notamment sur l'accompagnement de 30000 exploitations dans leur transition vers des systèmes agro-écologiques à faible dépendance en produits phytopharmaceutiques » (MAAF et MEDDE, 2015). L'objectif de baisse des IFT des autres fermes que celles du réseau DEPHY est ici affirmé. Mais ce changement d'échelle ne semble pas avoir été pensé par les pouvoirs publics autrement que sous la forme d'une simple diffusion «par-dessus la haie »: la simple mise en visibilité, via la communication, des pratiques permettant une réduction de l'usage devrait, d'après les responsables du plan, convaincre les agriculteurs de les adopter.

Peut-on espérer qu'avec le temps ce mécanisme suffise pour atteindre la baisse du NODU attendue ? On peut en douter. En premier lieu parce que, ainsi que le montrent Akrich et al. (1988), le destin d'une innovation dépend de la participation active de ceux qui sont décidés à la faire avancer : « Adopter une innovation, c'est l'adapter. » En rupture avec le modèle classique de diffusion des innovations de Schumpeter, Akrich et al. soulignent que l'adoption d'une innovation suppose un important travail collectif qui repose sur l'engagement actif de tous les acteurs impliqués, et notamment les « récepteurs» de l'innovation, les « adoptants». Or, l'étude Tercia note la très faible participation des agriculteurs aux journées de communication régionales : «Les ingénieurs réseau et les membres des groupes constatent que ces journées n'atteignent pas les cibles recherchées [...]. Elles attirent surtout les convaincus. Certains disent continuer à les organiser uniquement parce qu'elles sont une obligation » (TERCIA, 2014). De surcroît, réussir le défi d'une réduction ambitieuse de l'utilisation des pesticides ne se résume pas à mettre au point et faire adopter des systèmes de culture économes. Cela nécessite aussi d'intégrer la dimension territoriale : aménagements paysagers pour amplifier certaines fonctions écologiques de régulation par les auxiliaires, diversité des assolements pour limiter la prolifération des parasites, organisation de la confusion sexuelle au niveau d'îlots de parcelles (Encadré 1)... Autant d'aspects, nécessairement collectifs, très peu pris en charge par le plan actuel.

Au lancement du plan Ecophyto 2, DEPHY repose donc sur un réseau orienté vers une communication centrée sur la diminution des IFT à l'intérieur du réseau, dans un souci de justification de moyens humains et financiers. Pour ce faire, les animateurs de groupe, acteurs centraux du dispositif, ont vu leur mission initiale d'accompagnateurs du changement évoluer vers une mission de collecte de données pour alimenter une base nationale, utilisée en retour pour la communication institutionnelle. La généralisation de systèmes économes et performants (encore peu développés dans Ecophyto 1), en lien avec l'objectif de diminution du NODU de la ferme France, reste un impensé pour les pouvoirs publics. Si le plan Ecophyto 2 affiche l'ambition de généralisation, pour le moment rien n'est avancé sur la stratégie qui sera employée pour y parvenir. 




Fig. 2. Évolution, entre 2008 et 2012, de la proportion d'articles traitant de la réduction des pesticides, classés selon les voies de réduction mises en avant. Le nombre d'articles analysés, publiés dans 4 revues techniques nationales s'adressant aux agriculteurs (Cultivar, La France Agricole, Perspectives agricoles, Réussir Grandes Cultures) est indiqué en haut de la figure, pour chaque année.

Fig. 2. Evolution, between 2008 and 2012, of the proportion of articles dealing with pesticide reduction, published in four national technical magazines aimed at farmers (Cultivar, La France Agricole, Perspectives agricoles, Réussir Grandes Cultures). Articles are ranked according to the highlighted strategies to scale down pesticide.

Source : auteurs.

\section{Un verrouillage du système sociotechnique qui freine le développement des alternatives}

Cette logique de mise en œuvre du plan, que nous venons de décrire, ne peut être complétement comprise qu'en la resituant dans le contexte sociotechnique de l'usage des pesticides : la faible place de la reconception des systèmes de culture, l'évolution des objectifs du réseau DEPHY, ou les ambiguités concernant les indicateurs, peuvent être expliquées par l'existence d'un verrouillage sociotechnique impliquant une grande partie des acteurs du monde agricole.

\subsection{La logique d'utilisation des pesticides, de la production agricole aux filières}

La logique actuelle d'utilisation des pesticides a été décrite dès 1991, dans un article intitulé "Produire autrement " par Meynard et Girardin (1991). S'appuyant sur l'exemple du blé d'hiver, ces auteurs décrivaient les raisonnements techniques qui, en vue d'obtenir un rendement le plus élevé possible, associaient un semis dense et précoce, une alimentation azotée non limitante et une variété choisie pour sa productivité, rendant obligatoire l'utilisation de pesticides. Comme l'a souligné l'étude Ecophyto R\&D (2010), ces raisonnements sont toujours à l'œuvre. Schott et al. (2010) montrent aussi que la spécialisation des territoires et des exploitations, qui a entraîné un raccourcissement des rotations et une homogénéisation des mosaïques paysagères, a également contribué à une utilisation accrue des pesticides. De manière générale, pour maximiser la fréquence de retour des espèces réputées les plus rentables ainsi que leur productivité, les agriculteurs privilégient des options techniques qui, de fait, favorisent les ennemis des cultures et rendent indispensable le recours aux pesticides : ceux-ci sont devenus la " clef de voûte " des systèmes de culture (Meynard et Girardin, 1991).

Ce rôle clé joué par les pesticides est renforcé par le système de conseil (Compagnone et al., 2015 ; Bonnaud et al., 2012). Les agriculteurs sont principalement conseillés, au quotidien, par les entreprises qui commercialisent les intrants : c'est cette vente, en particulier celle des pesticides, qui leur permet de financer un conseil de proximité. On comprend bien qu'un conseil technique attaché à la vente d'intrants privilégie en général les solutions simples (un problème, un intrant) plutôt que les méthodes agronomiques préventives, plus complexes à mettre en œuvre et d'efficacité moins directe (Meynard, 2012). Un recensement des thèmes abordés dans les articles de la presse agricole nationale consacrés à la réduction des pesticides en grande culture confirme que la communication sur la réduction d'utilisation porte majoritairement sur l'amélioration des modalités de raisonnement des traitements, principalement par des « outils d'aide à la décision » (Fig. 2), plus que sur les moyens de prévention, en particulier la combinaison entre techniques complémentaires dans les systèmes de culture.

De fait, ainsi que le montrent les entretiens réalisés dans le cadre de l'étude Ecophyto R\&D (Meynard et al., 2010), les acteurs de la R\&D et du conseil s'intéressent prioritairement aux voies de réduction des pesticides qui n'obligent pas les agriculteurs et les filières agricoles à modifier plusieurs pratiques ou leur organisation. Cette étude a permis d'en recueillir de nombreuses illustrations. Par exemple, les variétés de pomme de terre résistantes au mildiou ne sont pas retenues par les industriels, car le changement de variétés nécessiterait un changement des process de transformation. Les associations de variétés de blé, efficaces pour réduire le recours aux fongicides, sont écartées par les coopératives et l'institut technique concerné parce que les meuniers préfèrent réaliser eux-mêmes leurs assemblages à partir de variétés pures. Les 
conduites de culture à « bas intrants » ne sont pas diffusées par ces mêmes acteurs, par crainte d'une réduction des volumes de collecte. « Les associations d'espèces ou la diversification des cultures, souligne l'étude Ecophyto $R \& D$, se heurtent à des contraintes de logistique au sein des organismes de collecte : la collecte d'une plus grande diversité de produits (diversification des espèces), et de produits aux caractéristiques très variables (associations d'espèces) semble aujourd'hui difficilement envisageable, par manque de silos et de cellules. » En analysant les « freins et leviers à la diversification des cultures », Meynard et al. (2015) complètent cette analyse : la diversification reste peu promue car les espèces de diversification n'ont souvent pas de débouché... en raison de leur faible surface qui ne permet pas d'assurer un approvisionnement régulier aux potentiels utilisateurs. Comme il n'y a pas de marché pour les semences de ces espèces, elles ne font pas l'objet d'un travail de sélection important, ce qui accroît leur handicap de productivité par rapport aux espèces dominantes, et restreint encore les chances que leur culture se développe.

\subsection{Un verrouillage autour des pesticides}

Les systèmes de production agricoles actuels, spécialisés et intensifs en intrants chimiques, apparaissent ainsi totalement cohérents avec l'organisation des filières amont et aval, et avec les systèmes de diffusion d'information. Tous les acteurs ont adapté leur stratégie, et leur relation aux autres acteurs, à l'existence de la solution "pesticides », et la stratégie de chacun renforce celle des autres. Personne n'a vraiment intérêt à remettre en cause les tendances lourdes auxquelles il s'est adapté. On se trouve dans une situation de "verrouillage sociotechnique » (Encadré 3) autour des systèmes de production spécialisés et intensifs en intrants chimiques, qui freine le développement des alternatives aux pesticides (Vanloqueren et Baret, 2008 ; Butault et al., 2010 ; Meynard et al., 2015).

Le verrouillage s'exerce dans de multiples dimensions à la fois, ce qui explique tant sa solidité que son emprise sur l'innovation (Meynard, 2012). Ainsi :

- au plan économique, l'amortissement des installations industrielles pousse à la spécialisation régionale des productions et au rejet des solutions techniques qui pourraient entraîner une baisse de la production, comme les itinéraires techniques à bas intrants ;

- au plan social, aucune organisation n'a de légitimité pour organiser, au niveau des territoires, la gestion collective que requièrent certaines alternatives aux pesticides (lutte biologique par conservation, gestion durable des résistances génétiques...) ; et il est plus risqué, pour la crédibilité d'un conseiller agricole, de se tromper en disant de ne pas traiter alors que ce serait nécessaire, qu'en conseillant de traiter alors que ce ne serait pas nécessaire (erreur qui passera souvent inaperçue) ;

- au plan cognitif, la familiarité des solutions simples (à chaque problème, son intrant) n'incite pas agriculteurs et conseillers à s'approprier les méthodes agronomiques préventives, vécues comme plus hasardeuses - et conduit de fait à une perte de compétences sur les solutions traditionnelles (rotations, semis différé...) ;

- au plan culturel, le prestige du rendement élevé et la représentation collective du « beau champ » (très vert et
Encadré 3 Le concept de verrouillage sociotechnique. Ce concept a été théorisé à partir du cas des claviers de machines à écrire (David, 1985), pour expliquer que la disposition des touches (QWERTY...) dictée par les contraintes mécaniques des premières machines perdure alors que d'autres claviers seraient plus ergonomiques. Des situations de verrouillage ont ensuite été mises en évidence dans de nombreux autres secteurs (énergie, transports). Le mécanisme général sous-jacent est que plus une technologie est adoptée, plus elle devient attractive et performante, par un phénomène d'autorenforcement lié à des effets de réseau, d'apprentissage, de norme, et à des économies d'échelle... Il en résulte qu'une technologie peut être adoptée de façon durable, voire irréversible, par la plupart des acteurs d'un système même si une technologie plus efficace apparaît. En fait, « une technologie n'est pas choisie parce qu'elle est la meilleure, mais elle devient la meilleure parce qu'elle a été choisie » (Arthur, 1989). Des situations de verrouillage ont été décrites en agriculture, pour expliquer les freins au changement des pratiques agricoles, notamment en vue de réduire l'usage des pesticides (Cowan et Gunby, 1996, aux États-Unis ; Vanloqueren et Baret, 2008, en Belgique ; Lamine, 2011 ou Fares et al., 2012, en France). Meynard et al. (2015) ont mis en évidence un verrouillage autour du raccourcissement des rotations et de la concentration de la production de grande culture sur un petit nombre d'espèces, qui entrave la diversification des assolements et contribue à consolider le verrouillage autour des pesticides.

homogène) chez les agriculteurs, et l'image du « beau fruit » (sans défauts extérieurs) chez les consommateurs, renforcent la dépendance aux pesticides ;

- au plan réglementaire, les autorisations de vente, accordées essentiellement aux variétés pures, font qu'un agriculteur ne peut trouver sur le marché des semences d'associations variétales; la normalisation de la qualité des fruits privilégie une absence de défauts de l'épiderme impossible à atteindre sans pesticides.

De très nombreux acteurs se trouvent donc solidairement impliqués dans ce verrouillage, et les agriculteurs ne disposent pas, à eux seuls, de leviers permettant une réduction ambitieuse de l'utilisation des pesticides. On peut penser que les résultats décevants du plan Ecophyto 1 sont liés, au moins en partie, à l'absence de mobilisation des acteurs de l'aval de l'agriculture (transformateurs, distributeurs, consommateurs), dont les stratégies et les spécifications de la demande contribuent de manière décisive au verrouillage. Mais aussi sans doute, au fait que les modalités du plan ont été concertées avec les représentants de la profession agricole majoritairement convaincus que le système sociotechnique actuel n'a pas vraiment d'alternative, et qui n'ont pas su se projeter dans un scénario de déverrouillage. De ce point de vue, rien ne semble avoir changé au moment du lancement du second plan, si l'on en croit « l'appel pour un Plan Ecophyto 2 pragmatique » 
émanant du Conseil de l'agriculture française (rassemblant les principales organisations professionnelles agricoles: la FNSEA, les Jeunes agriculteurs [JA], l'APCA, Coop de France), qui, contre toute évidence, argue de l'absence de solutions alternatives aux pesticides pour rejeter les objectifs et le plan du ministère. «Nous raisonnons les applications et sommes prêts à aller plus loin si nous disposons de moyens techniques et agronomiques pour le faire. [...] Le Gouvernement propose un projet de plan Ecophyto 2 qui ne permet pas, en l'état, de relever ce défi [...]. Ce plan doit fixer des objectifs réalisables en fonction des alternatives disponibles dans les cinq prochaines années. " (Conseil de l'agriculture française, 2015). Et il propose que la priorité soit « de développer la recherche et l'innovation opérationnelles pour identifier des solutions viables et innovantes ».

\section{Ecophyto 1 : un impact sur le plan symbolique?}

Le premier plan a eu peu d'impacts sur un plan opérationnel. Après cinq ans de mise en œuvre, l'échec est patent : ni l'objectif (les $50 \%$ ), ni même le cap (une tendance baissière) ne sont tenus. La réduction des pesticides se heurte en fait à une organisation collective extrêmement robuste et cohérente de l'agriculture, de ses filières et de sa recherchedéveloppement. Informer les agriculteurs, leur montrer l'intérêt des solutions alternatives, perfectionner celles-ci, ne saurait suffire. En adoptant, pour le changement de pratiques, une stratégie misant sur la diffusion par l'exemple, les concepteurs du plan ont sous-estimé l'ampleur des évolutions nécessaires, qui ne sont pas que techniques, mais aussi culturelles, cognitives, ou sociales; elles ne concernent pas que les parcelles, mais également les territoires et les filières. La force du verrouillage est de masquer aux acteurs concernés (ici les pouvoirs publics comme les agriculteurs ou les conseillers) l'existence même des voies alternatives au business as usual. Une baisse ambitieuse des pesticides devra passer par un « déverrouillage » qui ne pourra venir que d'une mobilisation simultanée et coordonnée de tous les acteurs concernés, c'est-à-dire de l'ensemble des acteurs de l'agriculture et de l'alimentation (consommateurs, transformateurs, distributeurs, producteurs, etc.). Une stratégie de réduction massive des pesticides devrait intégrer une vision systémique, prenant en compte les interdépendances liant l'ensemble des acteurs composant le système de production et de commercialisation propre à chaque filière agricole. Le plan Ecophyto 1 n'est pas entré dans cette logique : ciblant essentiellement les agriculteurs et la $R \& D$, il ne prévoyait rien pour inciter les sélectionneurs, les filières, les transformateurs, la grande distribution ou les consommateurs à changer leurs stratégies et leurs pratiques.

Les impacts du plan Ecophyto 1 ne sont pour autant pas négligeables. L'existence même du plan, en synergie avec d'autres actions de la politique de soutien à l'agroécologie, a encouragé les initiatives d'agriculteurs et de conseillers orientées vers l'invention d'une agriculture plus économe en intrants. Ceux-ci, malgré la pression sociale ou le manque de références locales, ont pu s'affirmer en s'organisant en groupes informels ou de type GIEE (groupements d'intérêt économique et environnemental), et échanger dans des réseaux internet. Dans une petite partie des groupes DEPHY, agriculteurs et conseillers ont progressé ensemble en produisant des références locales sur les systèmes de culture innovants. Ces évolutions sont restées minoritaires, puisqu'elles n'ont pas encore provoqué une baisse du NODU. L'ambition du plan Ecophyto, mais aussi son échec vis-àvis de la réduction du NODU, invitent à repenser l'accès des agriculteurs aux connaissances et l'organisation du développement agricole.

Ecophyto 1 a également eu un impact sur un plan symbolique. L'affirmation forte, par le ministère de l'Agriculture, de la nécessité de « produire autrement », en réduisant en particulier l'usage des pesticides, semble avoir modifié les visions de l'avenir et la définition des priorités des différents acteurs : par exemple, l'INRA a réorganisé son dispositif de recherche sur la « gestion intégrée de la santé des cultures »; les chambres d'agriculture et les CIVAM (centres d'initiatives agricoles) mettent en avant des compétences en accompagnement des agriculteurs dans le changement ; nous avons été frappés, lors de nos enquêtes récentes, de constater à quel point les acteurs les plus rétifs à cette politique de réduction de l'usage des pesticides reconnaissaient qu'elle marquait un changement de paradigme qui devait les inciter à terme à faire évoluer leur activité et leurs compétences, comme l'illustre cet extrait d'un entretien chez Bayer R\&D (24 avril 2015) :

« Nous on sait que l'époque n'est plus au tout pesticides, on en est bien conscient : quand vous voyez que des politiques publiques fixent le cap d'une baisse de moitié de l'usage des phytos, même si c'est irréaliste - on s'en aperçoit toujours plus, le ministre vient de l'avouer - ça donne le ton de la société. Il y a une demande de la société, et nous comme producteurs on doit y répondre. On est persuadé que la part du chimique sera de plus en plus décroissante ou en tout cas très ciblée. C'est en grande partie pour cela que l'on a créé en interne une division spécialisée sur la lutte biologique. »

Mais, comme le mentionne cette citation, les effets de cette « prise de conscience » pourraient se traduire surtout par la mise en avant de la « substitution » au travers des produits de biocontrôle. Il serait cependant dommage que la logique de retour sur investissements tende à favoriser uniquement la substitution, alors qu'on a aussi besoin d'un important travail de reconception des systèmes de culture, au niveau de la recherche comme du développement local.

La version 2 du plan révise les échéances : l'ambition de moins $50 \%$ est maintenue mais repoussée à 2025 , avec un objectif intermédiaire de moins $25 \%$ fixé pour 2020 . Ce nouveau plan met en avant la continuité, en renforçant les « actions structurantes » (BSV et réseau de fermes DEPHY notamment) de la version 1 , sans que les évolutions prévues ne permettent de répondre aux limites évoquées dans cet article. Par exemple, la rénovation du BSV se traduirait par un BSV enrichi de la présentation de méthodes alternatives, du rappel du besoin d'observations au champ et de mentions éventuelles plus explicites de la non-nécessité de traiter. Mais rien n'est évoqué par exemple quant à la structuration de l'échantillonnage à des fins diagnostiques. Concernant le réseau DEPHY, l'ambition du « facteur 10 » (travail avec 30000 fermes) est affirmée mais rien n'est dit sur les modalités de ce changement d'échelle. Le nouveau plan assoit sa stratégie sur des moyens qui relèvent essentiellement des deux stratégies 
d'efficience des traitements (grâce notamment au déploiement d'outils d'aide à la décision et au BSV) et de substitution de produits (le biocontrôle en est l'archétype), renvoyant à plus tard une approche plus systémique. Enfin, le nouveau plan n'explicite qu'une seule action ciblée sur des acteurs importants du verrouillage : sous forme expérimentale pour cinq ans, explorer la mise en ouvre de certificats d'économie de produits phytosanitaires (CEPP) auprès des distributeurs de pesticides. Cette approche rénovée est portée par une évolution des indicateurs de suivi, avec un accent très fort porté sur les moyens mis en œuvre au détriment des résultats.

Nous espérons que l'analyse et les propositions contenues dans cet article contribueront à aider les acteurs de l'agriculture, pouvoirs publics en tête, à infléchir leurs actions, et à se mobiliser collectivement pour surmonter la situation verrouillée que nous avons décrite. Les changements, parfois ténus mais réels, que l'on peut percevoir dès aujourd'hui chez une petite minorité d'agriculteurs, de conseillers, de coopératives ou d'entreprises d'amont et d'aval constitueront-ils l'amorce de la grande transition environnementale que le plan Ecophyto 2 entend favoriser ? Le choc symbolique occasionné par Ecophyto 1 prépare cette transition. Les attentes de la société, et les inquiétudes croissantes concernant la santé des agriculteurs et des consommateurs y poussent. Mais on n'échappera pas à la nécessité d'une réflexion approfondie sur le renouvellement des modalités de l'action publique, pour qu'elle réussisse à impulser des changements systémiques.

Remerciements. Cet article a bénéficié des travaux de recherche du projet "Pestimute-Gen » menés dans le cadre de l'appel à projet de recherche «Pour et sur le plan Ecophyto (PSPE) », action pilotée par le ministère chargé de l'agriculture avec l'appui financier de l'Office national de l'eau et des milieux aquatiques (Onema), par les crédits issus de la redevance pour pollutions diffuses attribués au financement du plan Ecophyto.

\section{Références}

Akrich M, Callon M, Latour B. 1988. A quoi tient le succès des innovations ? 1 : L'art de l'intéressement ; 2 : Le choix des porteparole. Gérer et comprendre. Annales des Mines 11: 4-17, 14-29. Disponible sur https://www.halshs.archives-ouvertes.fr/halshs00081741/document.

Arthur B. 1989. Competing technologies, increasing returns, and lock-in by historical events. Econ $J$ 99(394): 116-131. doi: 10.2307/2234208.

Aubertot JN, Barbier JM, Carpentier A, Gril JJ, Guichard L, Lucas S, et al. 2005. Pesticides, agriculture et environnement: réduire l'utilisation des pesticides et en limiter les impacts environnementaux. In : Expertise scientifique collective, synthèse du rapport. France : INRA et Cemagref, 64 p. Disponible sur http://www.inra. dam.front.pad.brainsonic.com/ressources/afile/234150-6a298resource-expertise-pesticides-synthese.html.

Blanchet P. 2012. Synthèse de l'évaluation in itinere de l'axe $5 \mathrm{du}$ plan Ecophyto 2018. In : CGAAER $n^{\circ}$ 10177. Ministère de 1'Agriculture. Disponible sur http://www.agriculture.gouv.fr/ sites/minagri/files/documents/pdf/Rapport_de_synthese_du_Presi dent du Comite d evaluation Axe 5 cle 4 c $8 \bar{d} 48$.pdf.

Bonnaud L, Bouhsina Z, Codron JM. $201 \overline{2}$. Le rôle du marché dans le contrôle des traitements phytosanitaires. L'exemple du secteur de la tomate. Terrains \& Travaux(1): 87-103. Disponible sur http:// www.cairn.info/revue-terrains-et-travaux-2012-1-page-87.htm.

Butault JP, Dedryver CA, Gary C, Guichard L, Jacquet F, Meynard JM, et al. 2010. Ecophyto R\&D. Quelles voies pour réduire l'usage des pesticides ? In : Synthèse du rapport d'étude. France : INRA, 90 p. Disponible sur http://www.inra.dam.front.pad.brainsonic. com/ressources/afile/224979-c9978-resource-ecophyto-r-d-syn these.html.

Cerf M, Veiga I, Prost L, Barcellini F. 2015. Designing for transition in agriculture: addressing the gap between design and innovation. In: Proceedings 19th Triennial Congress of the IEA, Melbourne, 914 August 2015.

Comité de la prévention et de la précaution. 2002. Risques sanitaires liés à l'utilisation des produits phytosanitaires. Ministère de l'Aménagement du territoire et de l'Environnement, $47 \mathrm{p}$. Disponible sur http://www.ladocumentationfrancaise.fr/var/sto rage/rapports-publics/024000113.pdf.

Comité d'orientation d'Ecophyto R\&D. 2010. Ecophyto R\&D. Quelles voies pour réduire l'usage des pesticides ? Cahiers d'acteurs. INRA, 67 p. Disponible sur http://www.inra.dam. front.pad.brainsonic.com/ressources/afile/224989-649ca-resourceecophyto-r-d-cahier-des-acteurs.html.

Compagnone C, Simon B, Moretty P. 2015. Coopération et concurrence entre organismes de conseil. Le cas du conseil phytosanitaire en Bresse Bourguignonne. In : Compagnone C, Goulet F, Labarthe P, eds. Conseil privé en agriculture : acteurs, pratiques et marché. Dijon/Paris : Educagri et Quae, p. 73-91.

Conseil de l'agriculture française. 2015. Appel pour un plan Ecophyto pragmatique. Disponible sur http://www.fnsea.fr/toutes-les-thema tiques/agriculture-durable/bonnes-pratiques-agricoles/articles/ appel-pour-un-plan-ecophyto-pragmatique/.

Cowan R, Gunby P. 1996. Sprayed to death: path dependence, lock-in and pest control strategies. Econ J 106: 521-542. doi: 10.2307/ 2235561.

David PA. 1985. Clio and the economics of qwerty. Am Econ Rev 75: 332-337. Disponible sur http://www.links.jstor.org/sici?sici=0002$8282 \% 28198505 \% 2975 \% 3$ A2\%3C332\%3ACATEOQ\%3E2.0.CO $\% 3$ B2-I.

Deguine JP, Gloanec C, Laurent JP. 2016. Protection agroécologique des cultures. Éditions Quae, 287 p.

Dreyfus F. 2014. Synthèse de l'évaluation in itinere du réseau DEPHY. In : CGAAER $n^{\circ}$ 121104. Ministère de l'Agriculture. Disponible sur http://www.agriculture.gouv.fr/sites/minagri/files/ documents/pdf/Note_synthese_evaluation_DEPHY_C GAAER vdef3 cle8da5e-7.pdf.

Dumas M, Moraine M, Reau R, Petit MS. 2012. FERME 2010 produire des ressources pour l'action à partir de l'analyse de systèmes de culture économes en produits phytosanitaires mis au point par les agriculteurs dans leurs exploitations. Tome I : méthodes et résultats. 72 p. + annexes. Disponible sur http://www. bourgogne.chambagri.fr/uploads/media/FERME_2010_Tome_I. pdf.

Ecophyto. 2015. Note de suivi 2015, tendances du recours aux produits phytopharmaceutiques de 2009 à 2014, 36 p. Disponible sur http://www.agriculture.gouv.fr/sites/minagri/files/20160301_note suivi_ecophyto2.pdf.

Fares M, Magrini MB, Triboulet P. 2012. Agroecological transition, innovation and lock-in effects: the impact of the organizational design of supply chains. Cah Agric 21: 34-45. doi: 10.1684/ agr.2012.0539.

Gliessman SR. 2007. Agroecology: ecological processes in sustainable agriculture, 2nd ed. Lewis publisher (CRC Press): Boca Raton, 408 p. doi:10.1017/S0014479707005364. 
Gravesen L. 2003. Reducing pesticide dependency in Europe to protect health, environment and biodiversity. In: PAN (Pesticide Action Network) Europe, Pure Conference 2003, Copenhagen.

InVivo. 2015. Communiqué de presse. Plan Ecophyto, InVivo salue des avancées majeures, mais souhaite aller plus loin dans son suivi. Disponible sur http:/www.invivo-group.com/sites/default/files/ atoms/files/plan-ecophyto-invivo-salue-des-avancees-majeuresmais-souhaite-aller-plus-loin.pdf.

Jacquet F, Butault JP, Guichard L. 2011. An economic analysis of the possibility of reducing pesticides in French field crops. Ecological economics(70): 1638-1648. doi: 10.1016/j.ecolecon.2011.04.003.

Lamine C. 2011. Transition pathways towards a robust ecologization of agriculture and the need for system redesign. Cases from organic farming and IPM. J Rural Stud 27: 209-219. doi: 10.1016/j. jrurstud.2011.02.001.

Le Breton M. 2014. Caractérisation de l'utilisation des pesticides en grandes cultures et en viticulture, amélioration de l'indicateur de fréquence de traitement phytosanitaire (IFT) et première déclinaison environnementale au regard de l'enjeu production d'eau potable, guide technique et algorithmes de calcul. Onema, Inra, Ineris, $210 \mathrm{p}$.

Le Corre N. 2011. Le réseau de fermes DEPHY Ecophyto. Préparation au 2e appel à candidature, $32 \mathrm{p}$.

Loyce C, Meynard JM, Bouchard C, Rolland B, Lonnet P, Bataillon P, et al. 2012. Growing winter wheat cultivars under different management intensities in France. A multicriteria assessment based on economic, energetic and environmental indicators. Field Crops Research 125(1): 167-178. doi: 10.1016/j.fcr.2011.08.007.

MAAF, MEDDE. 2015. Plan Ecophyto II, agroécologie, produisons autrement. 66 p. Disponible sur http://www.agriculture.gouv.fr/ sites/minagri/files/151022_ecophyto.pdf.

Meynard JM. 2012. Innovating in cropping and farming systems (chapter 5). In: Coudel E, Devautour H, Soulard CT, Faure G, Hubert B, eds. Renewing innovation systems in agriculture and food: how to go towards more sustainability? Wageningen Academic Publishers, p. 89-108.

Meynard JM, Girardin P. 1991. Produire autrement. Courrier de la cellule environnement de l'INRA 15: 1-19. Disponible sur http://www7.inra. fr/lecourrier//wp-content/uploads/2012/01/C15Girardin.pdf.

Meynard JM, Barbier JM, Bonicel L, Dubeuf JP, Guichard L, Halska J, et al. 2010. Ecophyto R\&D. Tome VII, analyse des jeux d'acteurs. 39 p. Disponible sur http://www.inra.dam.front.pad. brainsonic.com/ressources/afile/224986-1b2c9-resource-eco phyto-r-d-tome-vii.html.

Meynard JM, Messéan A, Charlier A, Charrier F, Fares M, Le Bail M, et al. 2015. La diversification des cultures : lever les obstacles agronomiques et économiques. Éditions Quae, 106 p.

Mischler P, Lheureux S, Dumoulin F, Menu P, Sene O, Hopquin JP, et al. 2009. Huit fermes de grande culture engagées en production intégrée réduisent fortement les pesticides sans baisse de marge. Le Courrier de l'Environnement 57: 73-91. Disponible sur http://www.prodinra.inra.fr/record/45047.
Momas I, Caillard JF, Lesaffre B. 2004. Rapport de la Commission d'orientation du Plan national santé-environnement, $252 \mathrm{p}$. Disponible sur http://www.ladocumentationfrancaise.fr/var/sto rage/rapports-publics/044000068.pdf.

Petit M-S, Reau R, Dumas M, Moraine M, Omon B, Josse S. 2012. Mise au point de systèmes de culture innovants par un réseau d'agriculteurs et production de ressources pour le conseil. Innovations agronomiques V 20 (2012): 79-100

Pillet E, coord. 2014. Réseau DEPHY-FERME. Synthèse des premiers résultats à l'échelle nationale. Ecophyto, ministère de l'Agriculture, Onema, 52 p. Disponible sur http://www.agriculture. gouv.fr/sites/minagri/files/documents/201411_Synthese_Resul tats_DEPHY_cle438e79.pdf.

Pinta P. 2015. Ecophyto 2 : une irresponsabilité frappante. Disponible sur http://www.agpb.com/actualit\%C3\%A9/ecophyto-2-une-irres ponsabilit\%C3\%A9-frappante, consulté le 29/8/2016.

Potier D. 2014. Pesticides et agroécologie, les champs du possible. In : Rapport au Premier ministre Manuel Valls. 202 p + annexes. Disponible sur http://www.dominiquepotier.com/UserFiles/File/ rapport-dpotier-pesticides-et-agro-ecologie-basse-def.pdf.

Reau R, Fortino G. 2010. Ecophyto R\&D, vers des systèmes de culture économes en produits phytosanitaires. Volet 2. Tome IX. Conception d'un réseau d'acquisition de références et d'un réseau d'information. 100 p. Disponible sur http://www.inra.dam.front. pad.brainsonic.com/ressources/afile/224988-6a203-resource-eco phyto-r-d-tome-ix.html.

Ricci P. 2015. Recherche et innovation dans le plan Écophyto : présentation synthétique du programme scientifique de l'axe recherche. Innovations Agronomiques 46: 157-169. Disponible sur http:/www.prodinra.inra.fr/ft?id=67A23A6A-67E8-4037-B3EDAA30F29E9357.

Salembier C, Elverdin JH, Meynard JM. 2016. Tracking on-farm innovations to unearth alternatives to the dominant soybean-based system in the Argentinean Pampa. ASD V 36: 1. doi: 10.1007/ s13593-015-0343-9.

Schott C, Mignolet C, Meynard JM. 2010. Les oléoprotéagineux dans les systèmes de culture : évolution des assolements et des successions culturales depuis les années 1970 dans le bassin de la Seine. OCL 17: 276-291. doi: 10.1051/ocl.2010.0334.

Soubeyran E. 2014. Exposé à l'Académie d'agriculture. Disponible sur https://www.academie-agriculture.fr/seances/les-systemes-decultures-economes-en-pesticides.

TERCIA-Consultants. 2014. Évaluation in itinere du réseau Dephy (axe 2, action 14 du plan Ecophyto). Paris : Onema ; ministère de l'Agriculture, de l'Agroalimentaire et de la forêt. 137 p. Disponible sur http:/www.agriculture.gouv.fr/sites/minagri/files/documents/ pdf/Tercia Evaluation DEPHY rapport final 05-022014_cle81919f.pdf.

Vanloqueren G, Baret P. 2008. Why are ecological, low-input, multiresistant wheat cultivars slow to develop commercially? A Belgian agricultural 'lock-in' case study. Ecological Economics 66: 436-446. doi: 10.1016/j.ecolecon.2007.10.007.

Citation de l'article : Guichard L, Dedieu F, Jeuffroy M-H, Meynard J-M, Reau R, Savini I. 2017. Le plan Ecophyto de réduction d'usage des pesticides en France : décryptage d'un échec et raisons d'espérer. Cah. Agric. 26: 14002. 